\title{
Clinical comparisons of $0.5 \%$ and $0.375 \%$ levobupivacaine for ultrasound-guided axillary brachial plexus block with nerve stimulation
}

\author{
Wonkyo Kim, Youn Jin Kim, Jong-Hak Kim, Dong Yeon Kim, Rack Kyung Chung, Chi Hyo Kim, and
} Seok Heo

Department of Anesthesiology and Pain Medicine, School of Medicine, Ewha Womans University, Seoul, Korea

Background: In an axillary brachial plexus block ( $\mathrm{ABPB}$ ), where relatively large doses of local anesthetics are administered, levobupivacaine is preferred due to a greater margin of safety. However, the efficacy of levobupivacaine in $\mathrm{ABPB}$ has not been studied much. We performed a prospective, double-blinded study to compare the clinical effect of $0.375 \%$ levobupivacaine with $0.5 \%$ levobupivacaine for ultrasound (US)-guided ABPB with nerve stimulation.

Methods: Forty patients undergoing elective upper limb surgery were randomized into two groups: Group I ( $0.375 \%$ levobupivacaine) and Group II (0.5\% levobupivacaine). All four main terminal nerves of the brachial plexus were blocked separately with $7 \mathrm{ml}$ of levobupivacaine using US guidance with nerve stimulation according to study group. A blinded observer recorded the onset time for sensory and motor block, elapsed time to be ready for surgery, recovery time for sensory and motor block, quality of anesthesia, patient satisfaction and complications.

Results: There were no significant differences in the time to find nerve locations, time to perform block and number of skin punctures between groups. Insufficient block was reported in one patient of Group I, but no failed block was reported in either group. There were no differences in the onset time for sensory and motor block, elapsed time to be ready for surgery, patient satisfaction and complications.

Conclusions: $0.375 \%$ levobupivacaine produced adequate anesthesia for ABPB using US guidance with nerve stimulation, without any clinically significant differences compared to $0.5 \%$ levobupivacaine. (Korean J Anesthesiol 2012; 62: 24-29)

Key Words: Axillary brachial plexus block, Levobupivacaine, Ultrasonography.

Received: February 8, 2011. Revised: 1st, April 1, 2011; 2nd, May 25, 2011; 3rd, May 27, 2011. Accepted: May 30, 2011.

Corresponding author: Youn Jin Kim, Ph.D., Department of Anesthesiology and Pain Medicine, School of Medicine, Ewha Womans University, 911-1, Mok-5 dong, Yangcheon-gu, Seoul 157-710, Korea. Tel: 82-2-2650-5285, Fax: 82-2-2655-2924, E-mail: ankyj@ewha.ac.kr

(c) This is an open-access article distributed under the terms of the Creative Commons Attribution Non-Commercial License (http:// creativecommons.org/licenses/by-nc/3.0/), which permits unrestricted non-commercial use, distribution, and reproduction in any medium, provided the original work is properly cited. 


\section{Introduction}

Axillary brachial plexus block (ABPB) is an anesthetic option commonly used for surgery of the hand, forearm, and elbow [1]. The conventional transarterial technique has potential problems such as nerve injury due to needle trauma and intraneural injection, as well as cardiovascular and CNS toxicity as a result of vascular uptake or accidental intravascular injection.

The use of ultrasound (US) has significantly improved the quality of nerve blocks by direct visualization of nerves and related anatomical structures, needle trajectory and spread of local anesthetics (LAs) during injection [2-7]. In addition, US guidance increases success rate, minimizes LAs volume needed for effective nerve block, and avoids potential complications such as intraneural or intravascular injection $[2,4,5]$.

Bupivacaine, ropivacaine and levobupivacaine are commercially available intermediate-acting LAs. They have some differences in risk of cardiovascular and CNS toxicity, but they have similar anesthetic and analgesic potency [8,9]. In $\mathrm{ABPB}$, in which relatively large doses of LAs are administered, ropivacaine and levobupivacaine are preferred due to a greater margin of safety.

There have been some studies on the efficacy of bupivacaine and ropivacaine in ABPB [10,11], and studies comparing levobupivacaine with bupivacaine or ropivacaine for neuraxial and other peripheral nerve blocks [9,12-14]. However, the efficacy of levobupivacaine in ABPB has not been studied much $[8,15]$. Therefore, we designed this study to compare the clinical effect of $0.375 \%$ levobupivacaine with $0.5 \%$ levobupivacaine for $\mathrm{ABPB}$ using US guidance with nerve stimulation.

\section{Materials and Methods}

After obtaining approval from the Institutional Review Board and written informed consent, forty American Society of Anesthesiologists (ASA) physical status I-II patients scheduled

Table 1. Demographic Data

\begin{tabular}{lcc}
\hline & Group I (n=20) & Group II (n=20) \\
\hline Age (yr) & $36.7 \pm 15.2$ & $40.7 \pm 13.2$ \\
Height (cm) & $165.3 \pm 10.5$ & $170.0 \pm 9.0$ \\
Weight (kg) & $65.4 \pm 12.3$ & $68.6 \pm 13.0$ \\
ASA physical status I/II (n) & $13 / 7$ & $12 / 8$ \\
Operation time (min) & $70.3 \pm 44.0$ & $81.3 \pm 50.2$ \\
Site of operation (n) & & \\
$\quad$ Hand & 11 & 6 \\
Wrist & 2 & 6 \\
Forearm & 7 & 8 \\
\hline
\end{tabular}

Data are mean \pm SD or number of patients. Group I: axillary brachial plexus block using $0.375 \%$ levobupivacaine. Group II: axillary brachial plexus block using $0.5 \%$ levobupivacaine. There were no significant differences between groups for any of these variables. for elective upper limb surgery, including forearm, wrist and hand procedures, were randomly allocated into two groups : Group I ( $\mathrm{n}=20$ ) received ABPB using 0.375\% levobupivacaine (Chirocaine $^{\circledR}$, Abbott Scandinavia AB, Solna, Sweden) and Group II $(\mathrm{n}=20)$ received ABPB using 0.5\% levobupivacaine. Patients with clinically significant coagulopathy, infection at the injection site, allergy to LAs, preexisting neuromuscular, cardiovascular, psychiatric and hepatic conditions, or age $<18$ years or $>65$ years were excluded. There were no significant differences in patient characteristics between groups (Table 1).

Patients were premedicated with $1-3 \mathrm{mg}$ of midazolam (Midazolam $^{\circledR}$, Bukwang Pharm, Seoul, Korea) intravenously. Standard monitoring, including noninvasive blood pressure, heart rate and pulse oximetry, was used throughout the procedure. Patients were placed in supine position with the operative arm abducted to $120^{\circ}$ and externally rotated with the forearm flexed to $90^{\circ}$.

Identification of the target nerve was performed using a SonoSite M-turbo (SonoSite ${ }^{\circledR}$, Bothell, WA, USA) with a 5-12 $\mathrm{MHz}$ linear transducer by one of the two investigators. The transducer was placed in a vertical orientation at the level of the anterior axillary fold, and the transducer was adjusted to place the axillary artery in the center of the image. Identification of each nerve was done by traceback approach technique. A 22-gauge, 50-mm short-beveled insulated needle (Stimuplex ${ }^{\circledR}$, B. Braun, Melsungen, Germany) was inserted and advanced via in-plane technique so that the needle shaft and tip could be visualized. The location of the target nerve was confirmed with the aid of a nerve stimulator (Stimuplex ${ }^{\circledR}$ Dig RC, B. Braun, Melsungen, Germany) simultaneously. At first, a motor response was sought by stimulating with a 0.6 to $0.8 \mathrm{~mA}$ current intensity and a frequency of $2 \mathrm{~Hz}$. After the proper twitch was elicited, stimulating intensity was progressively reduced to less than $0.5 \mathrm{~mA}$. Once the proper twitch was maintained with a current less than $0.5 \mathrm{~mA}, 1 \mathrm{ml}$ of LA was injected. After this injection stopped the twitch, the location was considered adequate, and the remaining $6 \mathrm{ml}$ was injected. All four main branches were blocked separately with $7 \mathrm{ml}$ of levobupivacaine (a total amount of $28 \mathrm{ml}$ ) according to the study group. The spread of LAs around the target nerve was evaluated and the needle tip position was continuously adjusted during injection to optimize the impregnation of nerve structure under real-time US guidance.

A blinded observer recorded the time to find nerve locations (i.e. the time from US application to localization of individual nerves), time to perform block (i.e. time from localization of individual nerves to completion of LAs injection) and number of skin punctures.

The degree of sensory block was assessed by pinprick in the relevant dermatome of the musculocutaneous nerve 
(forearm), radial nerve (dorsal $1^{\text {st }}$ and $2^{\text {nd }}$ intermetacarpal area), median nerve (palmar side of the tip of $3^{\text {rd }}$ finger), and ulnar nerve (palmar side of the tip of the $5^{\text {th }}$ finger), and was graded according to a two-grade scale $(0=$ no block, $1=$ loss of pinprick sensation). The degree of motor block was measured by assessing the following motor functions: elbow flexion for the musculocutaneous nerve; extension/supination of arm and finger for the radial nerve; flexion/pronation of wrist and $2-3^{\text {rd }}$ finger for the median nerve; $4-5^{\text {th }}$ finger flexion/thumb adduction for the ulnar nerve. The degree of motor block was graded according to a three-grade scale $(0=$ no block, $1=$ partial block, 2 = complete block). Sensory and motor blocks were assessed every 5 minutes after injection for 30 minutes.

Surgical anesthesia was defined as sensory block grade 1 and motor block grade 1 or 2 in all four nerves. When a patient achieved surgical anesthesia, the patient was assumed to be "ready for surgery". The elapsed time to be ready for surgery was recorded.

To compare the time to be "ready for surgery" between the two groups, degree of sensory and motor block were assessed in all patients for 30 minutes. At 30 minutes, the number of patients reaching each grade of block was counted. Patients who had achieved surgical anesthesia were transferred to the operating room, and those that had not achieved surgical anesthesia were monitored until they were ready. If surgical anesthesia was not achieved within 1 hour, the block was considered as a "failed block", and conversion to general anesthesia was made.

The quality of anesthesia was measured by need for opioid during operation. In case of pain more than 4 on a numerical rating scale or uncomfortable sensation developed during surgery, $50 \mu \mathrm{g}$ boluses of fentanyl (Fentanyl ${ }^{\circledR}$, Myung-moon Pharm, Seoul, Korea) were administered intravenously. If pain persisted 5 minutes after administration of $50 \mu \mathrm{g}$ fentanyl, an additional $50 \mu \mathrm{g}$ was given intravenously. If no intraoperative analgesia was required, the block was assumed to be a "complete block". When less than $100 \mu \mathrm{g}$ of fentanyl was needed to complete surgery, the block was assumed to be a "sufficient block". If more than $100 \mu \mathrm{g}$ of fentanyl was needed to complete surgery, the block was assumed to be an "insufficient block". If fentanyl supplementation was not sufficient to complete surgery, the block was considered a "failed block", and conversion to general anesthesia was made.

Any complications, such as paresthesia, hematoma, infection and LAs toxicity, were noted during block performance, in the perioperative period, and during the 24 hours after LAs injection.

After the end of surgery, patients were transferred to the recovery room and patient satisfaction was assessed using a two point scale : " 0 = good; if ever operated on again in the future,
I want the same anesthetic technique" and " 1 = bad; if ever operated on again in the future, I want a different anesthetic technique."

24 hours after LAs injection, complete recovery of sensory and motor block was checked.

Statistical analysis was performed using SPSS for Windows, version 17.0 (SPSS Inc, Chicago, IL, USA). All data were expressed as mean \pm SD or absolute numbers. Wilcoxon-MannWhitney test was used to compare the distributions of age, height, weight, operation time, time to find nerve locations, time to perform block, number of skin punctures, onset time for sensory and motor block, and elapse time to be ready for surgery. Chi-square test was used to determine if there was a relationship between ASA physical status, site of operation, need for intraoperative opioid, and patient satisfaction. A P value of less than 0.05 was considered statistically significant.

\section{Results}

There were no significant differences in the average time to find nerve locations, time to perform block and number of skin punctures between groups. There were no significant differences in sensory and motor block time to grade 1 between groups (Table 2).

At 30 min after injection of LAs, the percentage of patients who reached sensory block grade 2 (complete sensory block) in the musculocutaneous, radial, median and ulnar nerve dermatomes were $75 \%, 40 \%, 15 \%, 40 \%$ in group I, and $80 \%$, $40 \%, 30 \%, 60 \%$ in group II (Table 3 ). The number of patients who reached motor block grade 2 (complete motor block) in the musculocutaneous, radial, median and ulnar nerve were $70 \%$, $55 \%, 10 \%, 15 \%$ in group I, and $75 \%, 40 \%, 25 \%, 20 \%$ in group II (Table 3). There were no statistically significant differences between two groups. The average elapsed time to be ready for surgery was $32.5 \pm 20.6$ minutes and $21.1 \pm 12.0$ minutes respectively, but this was not significantly different (Table 2).

There was no significant difference in the quality of blockade between the groups (Table 2). One patient in group I required $100 \mu \mathrm{g}$ of IV fentanyl, but no failed block was reported in either group. No patient demonstrated any signs of LAs toxicity. Patient satisfaction was good in both groups (Table 2).

All patients reported complete recovery of sensory and motor function.

\section{Discussion}

With US-guided ABPB combined with nerve stimulation, our study showed that $28 \mathrm{ml}$ of $0.375 \%$ or $0.5 \%$ levobupivacaine for ABPB induced similar clinical efficacy, including the time to block onset, elapsed time to be ready for surgery, quality 
Table 2. Results of Axillary Brachial Plexus Block with Levobupivacaine

\begin{tabular}{lcc}
\hline & Group I ( $\mathrm{n}=20)$ & Group II (n=20) \\
\hline Time to find nerve location (min) & $1.6 \pm 0.7$ & $2.0 \pm 1.1$ \\
Time to perform block (min) & $6.5 \pm 3.1$ & $6.5 \pm 2.1$ \\
Skin puncture (n) & $1.5 \pm 0.5$ & $1.5 \pm 0.5$ \\
Sensory block time to grade 1 (min) & & $6.8 \pm 5.5$ \\
$\quad$ Musculocutaneous n. & $10.5 \pm 10.6$ & $12.8 \pm 10.8$ \\
Radial n. & $15.1 \pm 12.8$ & $14.3 \pm 14.8$ \\
Median n. & $15.4 \pm 12.1$ & $11.2 \pm 10.3$ \\
Ulnar n. & $18.0 \pm 15.8$ & $7.2 \pm 5.5$ \\
Motor block time to grade 1 (min) & $7.1 \pm 6.9$ & $11.6 \pm 9.6$ \\
Musculocutaneous n. & $9.8 \pm 8.9$ & $16.1 \pm 13.6$ \\
Radial n. & $24.0 \pm 19.6$ & $14.1 \pm 12.4$ \\
Median n. & $25.5 \pm 19.3$ & $21.1 \pm 12.0$ \\
Ulnar n. & $32.5 \pm 20.6$ & $0 / 20$ \\
Elapsed time to be ready for surgery (min) & $1 / 19$ & $20 / 0$ \\
Intraoperative opioid : need/need not (n) & $20 / 0$ & \\
Patient satisfaction: G/N & & \\
\hline
\end{tabular}

Data are mean \pm SD or number of patients. Group I: axillary brachial plexus block using $0.375 \%$ levobupivacaine. Group II: axillary brachial plexus block using $0.5 \%$ levobupivacaine. Sensory block was graded according to a three grade scale: $0=$ no block, $1=$ loss of pinprick sensation, $2=$ loss of touch sensation. Motor block was graded using a three grade scale: $0=$ no block, $1=$ partial block, $2=$ complete block. There were no significant differences between groups for any of these variables.

Table 3. Patients with Complete Block in Each Nerve Territory 30 Minutes after Injection

\begin{tabular}{lrr}
\hline \multicolumn{1}{c}{ Location } & Group I $(\mathrm{n}=20)$ & Group II (n=20) \\
\hline Complete sensory block & & \\
Musculocutaneous $\mathrm{n}$. & $15(75 \%)$ & $18(90 \%)$ \\
Radial n. & $8(40 \%)$ & $8(40 \%)$ \\
Median n. & $3(15 \%)$ & $6(30 \%)$ \\
Ulnar n. & $8(40 \%)$ & $12(60 \%)$ \\
Complete motor block & & \\
Musculocutaneous n. & $16(80 \%)$ & $15(75 \%)$ \\
Radial n. & $11(55 \%)$ & $8(40 \%)$ \\
Median n. & $2(10 \%)$ & $5(25 \%)$ \\
Ulnar n. & $3(15 \%)$ & $4(20 \%)$ \\
\hline
\end{tabular}

Data are expressed as number (\%). Group I: axillary brachial plexus block using $0.375 \%$ levobupivacaine, Group II: axillary brachial plexus block using $0.5 \%$ levobupivacaine. There was no significant differences between the groups.

of block as well as the percentage of patients with complete sensory block and complete motor block at 30 min after LAs injection.

The reasons why we compared the clinical effect of $0.375 \%$ levobupivacaine to $0.5 \%$ levobupivacaine in particular were: 1) $0.5 \%$ levobupivacaine is the drug of choice for regional anesthesia in our institute, 2) ABPB with low-dose LAs has advantage of reducing risk of LAs toxicity, 3) in order to achieve surgical anesthesia, however, the dose of LAs cannot be markedly lowered.

Levobupivacaine, the $\mathrm{S}(-)$-enantiomer of bupivacaine, has similar anesthetic and/or analgesic potency $[8,9,12]$ in addition to less cardiac and central nervous system toxicity $[16,17]$ compared to bupivacaine. Its duration of sensory block is longer than ropivacaine $[8,15]$, so it has the advantage in providing prolonged postoperative pain control. The use of low-dose LAs in $\mathrm{ABPB}$, where relatively large volume of LAs are indicated, also may reduce the risk of LAs toxicities. There have been some efforts to lower the dose of bupivacaine and ropivacaine in ABPB [11], but only a few studies with similar investigations with levobupivacaine in ABPB. Among many possible concentrations, we chose to use $0.375 \%$ levobupivacaine based on the findings of Cox et al. and Hickey et al. $[13,18]$ as well as our experience in regional anesthesia for upper limb surgery. Hickey et al. [18] compared $0.25 \%$ ropivacaine with $0.25 \%$ bupivacaine for supraclavicular block and concluded that the $0.25 \%$ concentration for brachial plexus block is not sufficient to achieve surgical anesthesia because of a slow onset and a high rate of inadequate block. Cox et al. [13] compared $0.25 \%$ or $0.5 \%$ levobupivacaine with $0.5 \%$ bupivacaine for supraclavicular block and reported that $0.25 \%$ levobupivacaine had a slower onset as well as a lower overall success rate than $0.5 \%$ levobupivacaine, although there were no significant differences.

In our study, complete sensory and motor blocks were achieved with only $28 \mathrm{ml}$ of LAs in nearly all patients, except for one patient in group I who needed $100 \mu \mathrm{g}$ of IV fentanyl intraoperatively. This high success rate seems to be due to the combined use of US/nerve stimulation, multiple-nerve infiltration and operator's skill.

Before the introduction of real time ultrasonographic guidance in regional anesthesia, block failures were mostly due to maldistribution of LAs or multiple septation within the axillary brachial plexus sheath $[19,20]$. These septations or fascial compartments serve to limit the spread of injected 
LAs around the brachial plexus nerve. The brachial plexus block guided by US was more effective than those guided by a nerve stimulator. US guidance for nerve localization significantly improved the success rate and quality of block, shortened the performance time of peripheral nerve blocks, and decreased potential complications such as intraneural and intravascular injection [2-7]. These improvements were mainly due to increased accuracy of needle positioning and direct visualization of diffusion of LAs during injection. Despite the advantages of real-time US application, ultrasonography alone is not perfect because ultrasonography is indirect, and allows for subjective interpretation by the individual operator. Therefore, we used a combination of US and nerve stimulation to attempt a $100 \%$ success rate with only $28 \mathrm{ml}$ of LAs, without untoward side effects. US-guided ABPB using nerve stimulation decreased the performance time and reduced complications compared to nerve stimulation alone [21-23].

In this study, multiple-injection technique was used to provide more effective anesthesia. Clinical studies have suggested that higher block success is achieved with multiple-injection technique than with single- or double-injection techniques $[24,25]$. With single-injection technique, success rate of ABPB varies from $54 \%$ to $80 \%$ [26] because the musculocutaneous and the radial nerve were insufficiently anesthetized with single-injection technique [27]. One explanation could be that the locations of the radial and musculocutaneous nerve are difficult to find. The ultrasonographic image of the radial nerve is covered by the acoustic shadow of the axillary artery. Further, the musculocutaneous nerve is separated from the other nerves at the level of the axilla and is located between the biceps and coracobrachial muscles. Another explanation could be that there are multiple septations within the axillary neurovascular sheath which interferes the diffusion of LAs $[19,20]$.

In our study, ABPB was performed by either an experienced anesthesia staff or one anesthesiology resident experienced in US-guided $A B P B$ with at least 20 procedures under supervision of the experienced anesthesia staff. Block performance by skilled operators may have contributed to the high success rate.

The percentage of patients who showed complete sensory block and complete motor block in each of the four innervation areas at 30 minutes after LAs injection was mostly below $50 \%$, except for the musculocutaneous nerve $(70-80 \%)$ and motor blockage of the radial nerve in group I (55\%). Our results are consistent with the study of Liisanantti et al. [8]; at 45 minutes after injection of $45 \mathrm{ml}$ of $0.5 \%$ levobupivacaine, complete sensory block and complete motor block (30\%) was less frequent than in the $0.5 \%$ ropivacaine $(67 \%)$ and $0.5 \%$ bupivacaine ( $47 \%$ ) groups. The low rate of complete block may be due to the low volume $(28 \mathrm{ml})$ used in our study.

Our mean performance time was within 10 minutes. This indicates that US-guided ABPB using nerve stimulation was not only safe, but also efficacious in busy operating situations compared to the simple transarterial technique. Short performance time is also closely related to patients' satisfaction.

In conclusion, when performing ABPB with $0.375 \%$ or $0.5 \%$ levobupivacaine, there were no significant differences in the clinical efficacy, including the time to block onset, quality of block and patient satisfaction. If we consider the fact that clinical doses of LAs can result in systemic toxicity, $0.375 \%$ levobupivacaine would be a reliable and safer choice for ABPB.

\section{References}

1. Schroeder LE, Horlocker TT, Schroeder DR. The efficacy of axillary block for surgical procedures about the elbow. Anesth Analg 1996; 83: 747-51.

2. Kapral S, Krafft P, Gosch M, Fleischmann D, Weinstabl C. Ultrasound imaging for stellate ganglion block: direct visualization of puncture site and local anesthetic spread. A pilot study. Reg Anesth 1995; 20: 323-8.

3. Grau T, Leipold RW, Conradi R, Martin E, Motsch J. Efficacy of ultrasound imaging in obstetric epidural anesthesia. J Clin Anesth 2002; 14: 169-75.

4. Marhofer P, Schrögendorfer K, Wallner T, Koinig H, Mayer N, Kapral S. Ultrasonographic guidance reduces the amount of local anesthetic for 3-in-1 blocks. Reg Anesth Pain Med 1998; 23: 584-8.

5. Marhofer P, Schrögendorfer K, Koinig H, Kapral S, Weinstabl C, Mayer N. Ultrasonographic guidance improves sensory block and onset time of three-in-one blocks. Anesth Analg 1997; 85: 854-7.

6. Kapral S, Greher M, Huber G, Willschke H, Kettner S, Kdolsky R, et al. Ultrasonographic guidance improves the success rate of interscalene brachial plexus blockade. Reg Anesth Pain Med 2008; 33: 253-8.

7. Sites BD, Beach ML, Spence BC, Wiley CW, Shiffrin J, Hartman GS, et al. Ultrasound guidance improves the success rate of a perivascular axillary plexus block. Acta Anaesthesiol Scand 2006; 50: 678-84.

8. Liisanantti O, Luukkonen J, Rosenberg PH. High-dose bupivacaine, levobupivacaine and ropivacaine in axillary brachial plexus block. Acta Anaesthesiol Scand 2004; 48: 601-6.

9. Ivani G, DeNegri P, Conio A, Grossetti R, Vitale P, Vercellino C, et al. Comparison of racemic bupivacaine, ropivacaine, and levo-bupivacaine for pediatric caudal anesthesia: effects on postoperative analgesia and motor block. Reg Anesth Pain Med 2002; 27: 157-61.

10. Vainionpää VA, Haavisto ET, Huha TM, Korpi KJ, Nuutinen LS, Hollmén AI, et al. A clinical and pharmacokinetic comparison of ropivacaine and bupivacaine in axillary plexus block. Anesth Analg 1995; 81: 534-8.

11. Bertini L, Tagariello V, Mancini S, Ciaschi A, Posteraro CM, Di Benedetto $\mathrm{P}$, et al. $\mathbf{0 . 7 5 \%}$ and $\mathbf{0 . 5 \%}$ ropivacaine for axillary brachial plexus block: a clinical comparison with $0.5 \%$ bupivacaine. Reg Anesth Pain Med 1999; 24: 514-8.

12. Kopacz DJ, Allen HW, Thompson GE. A comparison of epidural levobupivacaine $0.75 \%$ with racemic bupivacaine for lower 
abdominal surgery. Anesth Analg 2000; 90: 642-8.

13. Cox CR, Checketts MR, Mackenzie N, Scott NB, Bannister J. Comparison of S(-)-bupivacaine with racemic (RS)-bupivacaine in supraclavicular brachial plexus block. Br J Anaesth 1998; 80: 594-8.

14. Casati A, Borghi B, Fanelli G, Montone N, Rotini R, Fraschini G, et al. Interscalene brachial plexus anesthesia and analgesia for open shoulder surgery: a randomized, double-blinded comparison between levobupivacaine and ropivacaine. Anesth Analg 2003; 96 : 253-9.

15. González-Suárez S, Pacheco M, Roigé J, Puig MM. Comparative study of ropivacaine $0.5 \%$ and levobupivacaine $0.33 \%$ in axillary brachial plexus block. Reg Anesth Pain Med 2009; 34: 414-9.

16. Bardsley H, Gristwood R, Baker H, Watson N, Nimmo W. A comparison of the cardiovascular effects of levobupivacaine and rac-bupivacaine following intravenous administration to healthy volunteers. Br J Clin Pharmacol 1998; 46: 245-9.

17. Huang YF, Pryor ME, Mather LE, Veering BT. Cardiovascular and central nervous system effects of intravenous levobupivacaine and bupivacaine in sheep. Anesth Analg 1998; 86: 797-804.

18. Hickey R, Rowley CL, Candido KD, Hoffman J, Ramamurthy S, Winnie AP. A comparative study of $0.25 \%$ ropivacaine and $0.25 \%$ bupivacaine for brachial plexus block. Anesth Analg 1992; 75: 6026.

19. Franco CD, Rahman A, Voronov G, Kerns JM, Beck RJ, Buckenmaier CC 3rd. Gross anatomy of the brachial plexus sheath in human cadavers. Reg Anesth Pain Med 2008; 33: 64-9.

20. Klaastad Ø, Smedby O, Thompson GE, Tillung T, Hol PK, Røtnes JS, et al. Distribution of local anesthetic in axillary brachial plexus block: a clinical and magnetic resonance imaging study. Anesthesiology 2002; 96: 1315-24.

21. Williams SR, Chouinard P, Arcand G, Harris P, Ruel M, Boudreault D, et al. Ultrasound guidance speeds execution and improves the quality of supraclavicular block. Anesth Analg 2003; 97: 1518-23.

22. Chan VW, Perlas A, McCartney CJ, Brull R, Xu D, Abbas S. Ultrasound guidance improves success rate of axillary brachial plexus block. Can J Anaesth 2007; 54: 176-82.

23. Orebaugh SL, Williams BA, Kentor ML. Ultrasound guidance with nerve stimulation reduces the time necessary for resident peripheral nerve blockade. Reg Anesth Pain Med 2007; 32: 448-54.

24. Lavoie J, Martin R, Tétrault JP, Côté DJ, Colas MJ. Axillary plexus block using a peripheral nerve stimulator: single or multiple injections. Can J Anaesth 1992; 39: 583-6.

25. Rodríguez J, Taboada M, Del Río S, Bárcena M, Álvarez J. A comparison of four stimulation patterns in axillary block. Reg Anesth Pain Med 2005; 30: 324-8.

26. Urban MK, Urquhart B. Evaluation of brachial plexus anesthesia for upper extremity surgery. Reg Anesth 1994; 19: 175-82.

27. Lanz E, Theiss D, Jankovic D. The extent of blockade following various techniques of brachial plexus block. Anesth Analg 1983; 62: 55-8. 